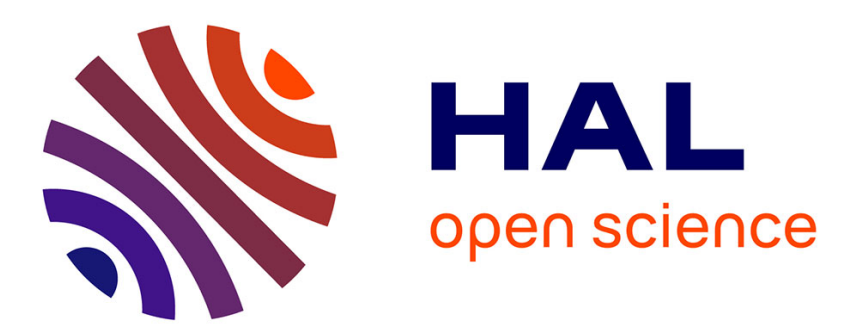

\title{
Institut Pasteur Minisymposium on Bacterial Membranes 2013
}

Nienke Buddelmeijer, Olivera Francetic

\section{To cite this version:}

Nienke Buddelmeijer, Olivera Francetic. Institut Pasteur Minisymposium on Bacterial Membranes 2013. Minisymposium on bacterial membranes, Mar 2013, Institut Pasteur [Paris], France. 10.1016/j.resmic.2013.06.006 . pasteur-01407699

\section{HAL Id: pasteur-01407699}

\section{https://hal-pasteur.archives-ouvertes.fr/pasteur-01407699}

Submitted on 2 Dec 2016

HAL is a multi-disciplinary open access archive for the deposit and dissemination of scientific research documents, whether they are published or not. The documents may come from teaching and research institutions in France or abroad, or from public or private research centers.
L'archive ouverte pluridisciplinaire HAL, est destinée au dépôt et à la diffusion de documents scientifiques de niveau recherche, publiés ou non, émanant des établissements d'enseignement et de recherche français ou étrangers, des laboratoires publics ou privés.

\section{(1)(1) $\$(0)$}

Distributed under a Creative Commons Attribution - NonCommercial - ShareAlikel 4.0 


\section{Accepted Manuscript}

Institut Pasteur Minisymposium on Bacterial Membranes 2013

Nienke Buddelmeijer, Olivera Francetic

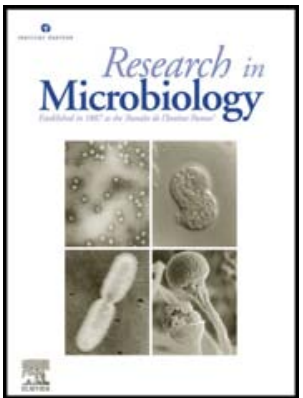

PII: S0923-2508(13)00107-1

DOI: $\quad$ 10.1016/j.resmic.2013.06.006

Reference: RESMIC 3206

To appear in: Research in Microbiologoy

Please cite this article as: $\mathrm{N}$. Buddelmeijer, O. Francetic, Institut Pasteur Minisymposium on Bacterial Membranes 2013, Research in Microbiologoy (2013), doi: 10.1016/j.resmic.2013.06.006.

This is a PDF file of an unedited manuscript that has been accepted for publication. As a service to our customers we are providing this early version of the manuscript. The manuscript will undergo copyediting, typesetting, and review of the resulting proof before it is published in its final form. Please note that during the production process errors may be discovered which could affect the content, and all legal disclaimers that apply to the journal pertain. 


\section{For publication}

Meeting report

\section{Institut Pasteur Minisymposium on Bacterial Membranes 2013}

The first Minisymposium on Bacterial Membranes was organized more than ten years ago and has become a successful tradition, with the 2013 edition taking place last March. This edition covered a variety of topics, including advances in the structural analysis of membrane proteins by solid-state NMR, the structure and function of components of several extracellular protein secretion systems, lipid modification and lipid-protein interactions, insertion of proteins into the outer membrane and uptake of DNA and nutrients. Over fifty young scientists from Germany, United Kingdom, Belgium and France gathered at the Institut Pasteur in Paris to share recent findings on these topics.

\section{Structural analysis of membrane proteins by solid-state NMR}

Structural analysis of membrane proteins is a prerequisite for understanding their function and biogenesis. Although membrane proteins form $30 \%$ of the proteome, they still represent only $1 \%$ of the structures in the Protein Data Bank. X-ray crystallography has been the main method used for structure determination. Typically produced in small quantities, requiring specific detergents for membrane extraction, flexible and dynamic, membrane proteins have been far fewer for yielding crystals of sufficient quality for X-ray diffraction analysis. Among alternative methods for structure determination, such as nuclear magnetic resonance (NMR) and electron microscopy (EM), solid-state NMR (ssNMR) has proven particularly powerful, providing structures of soluble and membrane proteins with equal success. Importantly, ssNMR is the only high-resolution technique that allows structural 
determination of protein polymers or aggregates, membrane proteins in native-like

27

environments or protein filaments / fibrils, without limitations in size and solubility. Thanks to a number of recent technical improvements, the limitations linked to the size of polymer subunits are progressively being reduced.

\section{Antoine Loquet (Max Planck Institute for Biophysical Chemistry, Göttingen,} Germany) described some of the most recently developed labeling techniques that greatly improve the resolution and information content of ssNMR data, using combinations of sparsely labeled carbon sources (Loquet et al., 2011). These approaches have proven extremely powerful when applied to structural analysis of the type III secretion needle from Salmonella typhimurium, revealing the molecular bases of stable polymer formation from flexible building blocks. Typically, polymer structures are deduced by rigid docking of isolated subunit structures into the electron density maps of multimers obtained by cryo-EM. These approaches are not only limited by the resolution of EM structures, but also by the extent of conformational changes that monomers undergo upon assembly within the polymer. Contrary to previous needle models obtained using rigid docking (Fujii et al., 2012), which placed the N-terminus of the monomer in the lumen of the needle, ssNMR analysis showed that these domains, disordered and invisible in X-ray monomer structures, adopt a rigid and defined conformation within the needle. An impressively detailed interaction network could be deduced from ssNMR data with over 150 intersubunit distance restraints. In the new model, the N-terminus of the needle subunit PrgI is stabilized by specific contacts with protomer $\mathrm{P}_{-11}$ and exposed on the needle surface (Loquet et al., 2012). This model was confirmed by immunogold labeling of the N-terminally tagged protein PrgI, not only on needles assembled in Salmonella, but also on needles of the Shigella T3SS formed by MxiH (Demers et al., 2013). 
Important components of NMR analysis are powerful computational methods, such as

53 ARIA or ISD, that allow structural calculations based on the distance data (Rieping et al.,

54 2008, Bardiaux et al., 2012). Since not only biological polymers, but also membrane proteins

typically exist as symmetric assemblies, the use of symmetry to calculate structures can

reduce calculation complexity and cost (Bardiaux et al., 2012). Benjamin Bardiaux (Institut

Pasteur, CNRS UMR3528, Paris, France) described the power of combining ssNMR and

computational methods based on ambiguous distance restraints to solve the structure of the

Yersinia adhesin A (YadA), secreted via the type V pathway (Leyton et al., 2012). YadA

belongs to a class of type $\mathrm{V}$ secretion systems called autotransporters, which cross the inner

membrane of Gram-negative bacteria via the Sec system. Their N-terminal part forms the

passenger domain that is secreted via a channel in the outer membrane formed by the C-

terminal beta-barrel domain. Cellular Bam machinery facilitates insertion of the C-terminal

domain into the outer membrane. Autotransporters are widespread and come in many flavors.

Yersinia YadA is a member of the trimeric autotransporter family, with the outer membrane

barrel formed by beta sheets derived from three polypeptides. The passenger domain of YadA has been analyzed by X-ray crystallography of its different fragments. Interestingly, poorly diffracting crystals of the outer membrane domain were used as material for ssNMR analysis, successfully providing the de novo structure of the trimer (Shahid et al., 2012). NMR analysis provided crucial information on the flexible nature of a short conserved tetrapeptide ASSA between the passenger and the beta-barrel domains. The results support the model where this sequence forms an essential hairpin that initiates secretion of the passenger domain, which is

73 subsequently driven by folding of the passenger domain into a beta-helix structure. 
establishment in the hostile niche of human gastric mucosa. Gaetano Castaldo (Vrije

77 Universiteit Brussels, Brussels, Belgium) uses X-ray crystallography to study the structure of 78 BabA, a 721 aa protein that binds to the Lewis b antigen (Yamaoka, 2008). Using a series of constructs facilitating BabA fragment production and purification, they revealed that these adhesins have an unusual fold. Although structural analysis is required to confirm this, preliminary biochemical studies strongly suggest that the BabA passenger domain is "inserted" into the beta-barrel-encoding polypeptide, thus defining a novel class of autotransporters.

\section{Type VI secretion: killing (or not) by secreted toxins}

86

Type VI secretion system (T6SS) analysis is currently one of the most dynamic areas of research in the field of bacterial protein transport. Although T6SSs are implicated in bacterial pathogenesis and interactions with eukaryotic host cells, their role in interspecies competition in the context of microbial communities and biofilms has been the focus of recent attention. Contact-dependent killing, discrimination between sister cells and members of different species and killing and resistance mediated by T6SSs are novel factors that need to be taken into account when considering seemingly peaceful communities of bacteria growing in close proximity on various surfaces. Yannick Brunet (University Aix-Marseille, Marseille, France) illustrated the role of T6SS in bacterial competition between two strains of the same species, the domesticated Escherichia coli $\mathrm{K}-12$ and a pathogenic enteroaggregative E. coli (EAEC). Co-cultivation of these strains results in killing of the laboratory workhorse in a contact-dependent manner. The "secret weapon" of the EAEC strains, the T6S apparatus, looks like an inverted phage tail with its piercing device pointing outwards. Assembled in the bacterial envelope, it is connected to a cytoplasmic shaft harboring a hollow tube filled with 
101 the panoply of "bullets", effector proteins with various enzymatic activities. Using live

102 fluorescence imaging and the GFP-labeled shaft component TssB, one can observe the 103 assembly and contraction of the shaft in a predator cell. This contraction in an EAEC cell that 104 is in direct contact with E. coli K-12 leads to the lysis of prey within minutes (Brunet et al., 105 2012). The tube is built as a stack of Hcp subunit hexamers, which can be assembled in vitro 106 both in a head-to-tail and tail-to-tail orientation. However, the preferred orientation in vivo is 107 head-to-tail, suggesting a mechanism that regulates tube assembly.

108 Serratia marcescens strain Db10 is a significant cause of hospital-acquired infections, 109 including in neonates. Using S. marcescens strain Db10 as model to study the T6SS function, 110 Grant English (University of Dundee, UK) focused on effector proteins secreted by this 111 system. Genome analysis identified genes encoding small highly acidic and highly basic 112 proteins that seem to come in pairs. They are non-essential for T6SS function and the basic 113 proteins Ssp1 and Ssp2 are indeed secreted by the T6SS. Interestingly, deletion of genes

114 encoding their acidic partners Rap1a and, in particular, Rap2a led to severe toxicity. Bacterial 115 filamentation caused by rap $2 a$ deletion was alleviated by T6SS inactivation or by deleting the 116 ssp2 gene, but not ssp1, strongly suggesting that these proteins act as toxin and immunity 117 protein pairs. Consistent with this model, purified Ssp1 and Rap1a form a stoichiometric 2:2 118 complex with nanomolar affinity. Remarkably, despite similar charge properties of these 119 pairs, binding is highly specific, since Ssp1 does not form a complex with Rap2a (English et 120 al., 2012). While structural analysis of other members of this class Rap1b and Rap2b provides 121 possible clues for this specificity, co-crystal structures of the cognate pairs should shed more 122 light on the mechanism by which antitoxins block the active sites as well as on toxin 123 activities. Recent in vitro studies show that Ssp1 is a member of cell-wall-degrading 124 amidases. Its production in the bacterial cytoplasm is tolerated, but its periplasmic production 125 leads to cell death, which is prevented by co-expression of its partner gene rapla. Similar 
126

127

128

129

130

131

132

133

134

135

136

137

138

139

140

141

142

143

144

145

146

147

148

149

150

results were obtained with the Ssp2 and Rap2a pair and several other toxin-antitoxin modules were found not only in the Db10 genome, but also in other species, including Agrobacterium and Salmonella. These results have interesting implications for the evolution of strains in populations, with continuous acquisition of toxin/antitoxin pairs with different specificities and enzymatic activities, contributing to rapid target killing, but also possibly propagating the same weapons and resistance proteins across species.

\section{Lipid modification and lipid-protein interactions}

Bacterial membranes are composed of phospholipids, proteins and modified lipids such as lipoteichoic acids (LTA), lipopolysaccharides (LPS) and lipoarabinomannan (LAM). Peter Bond (University of Cambridge, Cambridge, UK) provided structural and thermodynamic insights into the interaction between the lipid moiety of LPS and the MD-2 co-receptor of Toll-like receptor 4 (TLR4) using a molecular dynamics simulation approach (Paramo et al., 2013). Molecular simulation is based on descriptions of energy states of protein structures obtained from X-ray and NMR data (i.e. the TLR4 co-receptor MD-2) in a specific environment, here an asymmetric lipid bilayer composed of phospholipids and LPS. These energy descriptions are translated into forces and then into motion, resulting in a dynamic structural movie of a protein in a membrane. MD-2 binds the lipid A moiety of LPS (Park et al., 2009). The simulation data presented by Bond showed that MD-2 induces stronger binding of the agonist (LPS) by TLR4 and that the conformational changes predicted in MD-2 depend on the fatty acids of LPS. Sabina Chalabaev (Institut Pasteur, Paris, France) discussed the regulation of lipid A modification in biofilm-grown bacteria. Different growth conditions have been shown to lead to alterations in the composition of the bacterial cell envelope. For example, bacteria in biofilms are more resistant to antimicrobial agents and 
151 their surface exposed polysaccharides differ in structure compared to free-living planktonic

152 bacteria (Ciornei et al., 2010). In E. coli biofilms, the lipid A moiety of LPS is modified by

153 addition of palmitate, a reaction catalyzed by PagP. This phenomenon is also observed in

154 other biofilm-forming enterobacteria. Activation of pagP expression occurs via SlyA, which

155 releases the inhibitory effect of $\mathrm{H}-\mathrm{NS}$ on expression of the pagP gene. The PhoPQ and

156 EvgAS pathways are not involved in induction of pagP expression. Muriel Masi (University

157 Paris XI, Orsay, France) presented results of her work on the molecular basis of recognition

158 of sugar entities within the cell wall of Corynebacterium glutamicum by mycoyltransferase A

159 (MytA). The cell envelope of Corynebacterineae is a complex structure containing lipids, 160 peptidoglycan and arabinogalactan that is itself covalently linked to mycolic acids, the so-

161 called mAGPG. Preliminary structural data on the N-terminus of MytA indicates the presence

162 of an esterase fold, while its C-terminus contains several unique repeats involved in mycolic

163 acid transfer to the sugars of the cell envelope. Masi et al. reported a surprising observation

164 that MytA interacts with peptidoglycan and not arabinogalactan, and that arabinogalactan

165 inhibits binding of peptidoglycan by MytA.

166

167 4. Transport of folded proteins across membranes

168

169

170

171

172

173

174

175

Proteins are synthesized in the cytoplasm as linear polypeptides and are typically exported across the inner membrane in an unfolded state through narrow membrane channels, minimizing chances of causing proton leakage that would be detrimental to the energetic state of the cell. An example is the inner membrane channel formed by the essential protein SecY with a constriction and a plug, or the more complex trans-envelope channels in type I, type III and type IV protein secretion systems. However, bacteria have also met the challenge of transporting folded proteins across the inner or outer membrane. In the late 1990s, one of the 
176 first Symposia on Bacterial Membranes witnessed the discovery of a novel transport system

177 allowing export of folded proteins, typically those that acquire various cofactors upon 178 synthesis in the cytoplasm (Santini et al., 1998). Such proteins are synthesized with N179 terminal signal sequences containing a double arginine sequence in a conserved signature 180 motif, lending the name to this export pathway-- the twin arginine translocation (Tat) 181 pathway. Three essential protein components of this export system, TatA, TatB and TatC, 182 have been extensively characterized (Palmer and Berks, 2012). TatA and TatB are single-span 183 membrane proteins with cytoplasmic membrane associated alpha-helical domains. TatC is a 184 polytopic membrane protein that is involved in specific interactions with Tat signal peptides. 185 Sarah Rollauer (University of Oxford, Oxford, UK) described the 3-D structure of TatC from 186 a themophillic bacterium Aquifex aeolicus, recently determined by X-ray crystallography 187 (Rollauer et al., 2012).

188 The TatC structure is a product of extensive groundwork employed to optimize the 189 expression and solubilization of the protein using an elegant TatC-GFP fusion tool to 190 facilitate the screening process. TatC has 6 transmembrane segments and a large surface area 191 with a concave face oriented towards the cytoplasm. To assess its binding to other Tat 192 components, cysteine cross-linking data provided information on putative interaction sites 193 with TatA and TatB. The dynamic nature of these interactions, however, makes it difficult to 194 provide a clear model of this complex in action or to understand how the translocation 195 proceeds without compromising the membrane impermeability. Nevertheless, together with in 196 vitro binding assays and a vast collection of mutations of E. coli TatC, the structure provides 197 molecular insight into the mechanism of signal sequence recognition and binding. Clearly, 198 this structure is an essential and crucial step forward in structure-function analysis of this 199 fascinating transport system. 
Type II secretion systems (T2SSs) provide a unique route for prefolded proteins to the

201 bacterial cell surface or to the surrounding medium, through an outer membrane pore formed

202 by the protein secretin. This unique class of membrane proteins forms multimeric channels

203 that are also present in bacteria producing filamentous phage and type III secretion systems.

204 One of the best studied is the family of T2SS secretins exemplified by the E. coli GspD and

205 Klebsiella oxytoca PulD. Domains N0, N1 and N2 residing in the periplasm have been

206 crystallized, providing insight into the molecular basis of their flexible nature. However, little

207 is known about the highly conserved module composed of domains N3 and C that seems to

208 contain all information required for formation of multimeric channels, their gating and

209 insertion into the outer membrane (Korotkov et al., 2011). While cryoEM has provided a

210 global view of secretins, a high-resolution structure of the channel is still lacking. Even if it

211 were available, structural information is unlikely to be sufficient to allow us to understand the

212 biogenesis of this multimer and its membrane insertion. Gerard Huysmans (Institut Pasteur,

213 Paris, France) described new biophysical approaches used to characterize the folding pathway

214 of PulD from the pullulanase type II secretion system. PulD is a $65 \mathrm{kDa}$ protein that forms

215 multimers in an in vitro transcription-translation system and inserts into liposomes apparently

216 without any accessory factors. Adding liposomes to the in vitro reaction allows one to

217 monitor the kinetics of multimer formation. Assays of resistance to SDS, urea and trypsin are

218 used to discriminate between the formation of different states along the folding pathway. This

219 analysis suggests that multimer formation precedes insertion of PulD into membranes.

220 Changing the composition of the bilayer by variation in the acyl chain length and saturation

221 and the reaction temperature allows one to manipulate the rate-limiting steps. Specific PulD

222 mutations that influence some of these steps have also been very useful tools for monitoring

223 the folding pathway of PulD. Interesting future directions of this work include structural or 
224 biophysical analysis of PulD variants blocked at different steps of this unique folding 225 pathway.

226

227

5. Uptake of DNA and nutrients

228

Although bacteria lack the ability to carry out endocytosis, a variety of molecules of different size and composition can enter bacterial cells utilizing specific transport machineries in the cell envelope. The Com system required for DNA uptake by the Gram-positive organism Streptococcus pneumoniae includes putative surface pili, a DNA translocation system in the membrane and an endonuclease EndA peripherally attached to the external side of the membrane. In this bacterium, competence for DNA transformation occurs during a narrow time span at the beginning of the exponential phase of growth. Using a fusion protein between EndA and YFP, Nathalie Campo (CNRS-LMGM, P. Sabatier University, Toulouse, France) showed that EndA-YFP is uniformly distributed in the membrane of non-competent

238 cells and concentrates into one or two foci located at mid-cell during competence. 239 Relocalization is dependent on membrane-bound DNA-receptor ComEA. They developed an 240 elegant method based on binding of fluorescently labelled (Cy3) PCR products to 241 demonstrate that DNA binding also takes place at midcell. Furthermore, a synthetic

242 fluorescent DNA fragment composed of cell division gene $f t s Z$ and $g f p$ was shown to bind at 243 midcell, was taken up by the cell, recombined into the chromosome at the fts $Z$ locus and 244 expressed with detection of the resulting functional FtsZ-GFP fusion protein. The process 245 was completed 70 minutes after addition of DNA.

246 Laurent Guillon (University of Strasbourg, Illkirch, France) also used fluorescence

247 microscopy to localize the biosynthetic machinery for the Pseudomonas aeruginosa 248 pyoverdine and to identify the site where this siderophore enters the cell following iron 
249 binding. Using fluorescent proteins fused to pyoverdine biosynthesis enzymes PvdA and

250 PvdQ, Guillon showed different localization sites for pyoverdine synthesis: at the old cell

251 pole and throughout the periplasm. Recent findings suggest that uptake of iron-bound

252 pyoverdine most likely occurs over the entire cell surface and not at a specific cellular 253 location.

\section{Concluding remarks}

Bacterial membranes, membrane proteins and cell surface structures involved in bacterial interactions with the environment and their hosts have been an important and 258 dynamic area of research. To understand how bacteria acquire their shape, communicate with their environment and impact the ecosystems, whether in an infected host or in a particular environmental niche, an ever-increasing number of tools are available for biochemical,

261 genetic, structural, visual, biophysical and bioinformatic analysis. Increasingly inter-

262 disciplinary and structure-oriented, the studies of membrane phenomena have provided 263 mechanistic insights, but also novel biological concepts. Initiated in the late 1990s, the

264 Minisymposia on Bacterial Membranes continue to foster informal exchanges in these areas and build ties between European groups mastering diverse experimental tools but sharing common interests. Over the years, these meetings have witnessed the substantial development of a dynamic scientific community and have favored its renewal.

\section{Acknowledgements}

We thank Tony Pugsley for initiating the Minisymposia on Bacterial Membranes and 


\section{References}

280 Bardiaux, B., Malliavin, T., Nilges, M., 2012. ARIA for solution and solid state NMR. 281 Methods Mol Biol.831,453-483.

282 Brunet, Y., Espinosa, L., Harchouni, S., Mignot, T., Cascales, E., 2012. Imaging Type VI 283 Secretion-Mediated Bacterial Killing. Cell Reports 3,36-41.

284 Ciornei, C., Novikov, A., Beloin, C., Fitting, C., Caroff, M., Ghigo, J., Cavaillon, J., Adib-

285 Conquy, M., 2010. Biofilm-forming Pseudomonas aeruginosa bacteria undergo 286 lipopolysaccharide structural modifications and induce enhanced infammatory cytokine 287 response in human monocytes. Innate Immun.16, 288-301.

288 Demers, J., Sgourakis, N., Gupta, R., Loquet, A., Giller, K., Riedel, D., Laube, B., Kolbe, B., 289 et al., 2013. The Common Structural Architecture of Shigella flexneri and Salmonella 290 typhimurium Type Three Secretion Needles. PLOS Pathog. 9, e1003245.

291 English, G., Trunk, K., Rao, V., Srikannathasan, V., Hunter, W., Coulthurst, S., 2012. New 292 secreted toxins and immunity proteins encoded within the Type VI secretion system gene 293 cluster of Serratia marcescens. Mol. Microbiol. 86, 921-936.

294 Fujii, T., Cheung, M., Blanco, A., Kato, T., Blocker, A., Namba, K., 2012. Structure of a type 295 III secretion needle at $7-\AA$ resolution provides insights into its assembly and signaling 296 mechanisms. Proc. Natl. Acad. Sci. U.S.A.109,4461-4466.

297 Korotkov, K., Gonen, T., Hol, W., 2011. Secretins: dynamic channels for protein transport 298 across membranes. Trends Biochem. Sci.36,433-443. 
299 Leyton, D., Rossiter, A., Henderson, I., 2012. From self sufficiency to dependence:

300 mechanisms and factors important for autotransporter biogenesis. Nat. Rev.

301 Microbiol.10,213_225.

302 Loquet, A., Lv, G., Giller, K., Becker, S., Lange, A., 2011. 13C spin dilution for simplified 303 and complete solid-state NMR resonance assignment of insoluble biological assemblies. J. 304 Am. Chem. Soc.133,4722-4725.

305 Loquet, A., Sgourakis, N., Gupta, R., Giller, K., Riedel, D., Goosmann, C., Griesinger, C., 306 Kolbe, M., et al., 2012. Atomic model of the type III secretion system needle. Nature $307 \quad 486,276-279$.

308 Palmer, T., Berks, B., 2012. The twin-arginine translocation (Tat) protein export pathway. 309 Nat. Rev. Microbiol.10,483-496.

310 Paramo, T., Garzon, D., Holdbrook, D., Khalid, S., Bond, P., 2013. The simulation approach 311 to lipid-protein interactions. Methods Mol. Biol.974,435-455.

312 Park, B., Song, D., Kim, H., Choi, B., Lee, H., Lee, J., 2009. The structural basis of 313 lipopolysaccharide recognition by the TLR4-MD-2 complex. Nature 458,1191-1195.

314 Rieping, W., Nilges, M., Habeck, M., 2008. ISD: a software package for Bayesian NMR 315 structure calculation. Bioinformatics 24,1104-1105.

316 Rollauer, S., Tarry, M., Graham, J., Jääskeläinen, M., Jäger, F., Johnson, S., Krehenbrink, M., 317 Liu, S.-M., et al., 2012. Structure of the TatC core of the twin-arginine protein transport 318 system. Nature 492,210-215.

319 Santini, C., Ize, B., Müller, M., Giordano, G., Wu, L., 1998. A novel sec-independent 320 periplasmic protein translocation pathway in Escherichia coli. EMBO J. 17,101-112.

321 Shahid, S., Bardiaux, B., Franks, W., Krabben, L., Habeck, M., van Rossum, B.-J., Linke, D., 322 2012. Membrane-protein structure determination by solid-state NMR spectroscopy of 323 microcrystals. Nature Methods 9,1212-1217. 
324 Yamaoka, Y., 2008. Roles of Helicobacter pylori BabA in gastroduodenal pathogenesis.

325 World. J. Gastroenterol.14,4265-4272.

326

327

328

329

330

331

332
Nienke Buddelmeijer*, Olivera Francetic

Institut Pasteur, Unité de Génétique moléculaire, CNRS ERL3526, 25 rue du Dr Roux F-75724 Paris Cedex, France

* Corresponding author E-mail address: niebud@pasteur.fr 